\title{
The Study of RTK Receiver Based on WIFI Hotspot in Wireless Communication Datalink
}

\author{
Yang Guo-dong \\ College of Geoexploration Science and Technology \\ Jilin University \\ Jilin, China \\ ygd@jlu.edu.cn
}

\author{
Li Zhao-tong * \\ College of Geoexploration Science and Technology \\ Jilin University \\ Jilin, China \\ 1258899623@qq.com
}

\begin{abstract}
The rise of 3G/4G mobile communications, WiFi hotspots and other wireless communication technology has a serious of advantages including low radiation, wide broadband, long communication distance, low power consumption and etc. Then here comes a question, whether can we achieve a new generation RTK positioning model making use of this advanced communication measure instead of traditional digital radio transmission one? According to the above technical analysis, with WiFi accessing to the surrounding WiFi hotspot or the temporary WiFi hotspot constructed by 3G/4G mobile communications, in the article, the author put the satellite positioning receiver with the functions of WiFi wireless networking, BDS and GPS dynamic precision differential (RTK) accesses into JLCORS, then achieve carrier phase correction parameters which are located by RTK, BDS, and GPS satellite, and further centimeter-level positioning. Detailed discussions are based on RTK receiver research and design which are about WiFi hotspots wireless communications datalink.
\end{abstract}

Keywords-WiFi hotspots, satellite positioning, RTK, wireless communications datalink, JLCORS

\section{INTRODUCTION}

To obtain RTK differential correction value information the raditional RTK (Such as Trimble R10,Leicavivo,Stonex S9, Hi-Target v30,ect) receiver using conventional RTK receiver cannot leave the built-in UHF high-freguency radio and built-in GPRS or CDMA phone module, making the instrument must be considered in the design of the physical shell size, power consumption, voltage matching, logic control circuitry, underlying software programs, customer demand characteristics, various countries and regions communication standard and emission limits[1]. For a model of RTK products, it leads to a situation that all manufacturers have to make a combining scheme which are dozens of designs, assembly, testing, software, price and detection of maintenance[2].

The research aims to gather various parts of communication function to a whole, function forwarding, take the choice of combination scheme to customer, adjust measures to local conditions, that will be greatly increase the reliability, consistency, universality and compatibility of the RTK products. Specifically, it takes the global unified differential, complicated communications for RTK to WiFi globally recognized communication protocols and standards, and then customers or marketing partners according to their respective countries, regions, laws and regulations or customer specific requirements can the choice flexible way access to the secondary communication of WiFi. If RS232 serial communication function of VHF, UHF Digital Radio, GPRS or CMDA module interfaces, WIFI hotspots, that means through the WiFi network communication technology can achieve only with WiFi interface receiver to realize remote CORS station or user access of base station construction, surveying and mapping and achieving cm-level real-time control[3][4].

With WiFi, traditional radio receiver module and the wireless mobile communication module, RTK satellite positioning terminal cannot be considered during design, so that the design, assembly, testing, and maintenance will become very simple. Of course, another advantage is cost greatly reduced.

\section{PRINCIPLE OF MEASUREMENT}

\section{A. RTK Technology}

As a new development and breakthrough of satellite positioning system, RTK has played a key role in today's urban measurement and construction, what is more, its development prospects are very impressive. Positioning accuracy of RTK which can be achieved that offering three-dimensional positioning results in the specified coordinate system, and its accuracy achieves centimeter is also higher than the GPS single point positioning[5][6].

The technology of RTK positioning system is composed of workstations and base stations, is connected by the wireless data. So the top priority thing of RTK is to build a wireless data link effectively. In the real time kinematic positioning, the first thing is to establish a base station to receive satellite signals continuously and observate satellite all along. Each moving station will match a hand book which can receive observation data of base station by wireless communications equipment all the time. Using the relative positioning of the hand book, calculating the 3d coordinate and measurement precision of the rover. According to the baseline calculating results gained by hand book, the user will know the quality of observation data, greatly improve the observation efficiency, reduce duplication of observation and the redundant observation, save manpower and material resources, economic and environmental protection. The RTK positioning measurement is divided into 
two kinds, rapid static positioning and dynamic positioning respectively, for different forms of production practice the combination of two measurement model will do the actual measurement process[7][8].

\section{B. JLCORS Technology}

CORS(Continuous Operational Reference System),is a technology blend of satellite positioning technology, wireless communication technology, computer processing technology, and other technical means, which also build run continuously tracking station in a fixed area, to provide continuous, uninterrupted, high-precision space location services as well as a variety of different information service for users[9][10]. JLCORS is composed by the reference station network, the control center, data center, the user of application system and digital communication system. JLCORS network system spreads all over Jilin province, is a star network that take center node as the control center[11].

\section{WiFi hotspots technology}

The full name of WiFi is Wireless Fidelity, also known as the $802.11 \mathrm{~b}$ standard, which is similar to the mobile network, and it can achieve wireless fidelity, connecting with various terminals in a wireless communications datalink way[12]. WiFi is similar to the mobile network .Compared with the laying wired network, WiFi networks has advantages of low-cost and convenience, comparing with the existing Bluetooth technology, the coverage of $\mathrm{WiFi}$ is broader and its propagation speed faster, as a result, currently it has been widely used in daily life and production. It can spread data effectively and in a high-speed in a longer propagation distance. The bandwidth is up to $11 \mathrm{Mbps}$, if the signal is weak or is affected by some of the outside world, the bandwidth can be adjusted to $5.5 \mathrm{Mbps}, 2 \mathrm{Mbps}, 1 \mathrm{Mbps}$ automatically, so that the reliability and stability of the network have a certain assurance. The main characteristics of WiFi hotspots is strong stability, high reliability and spread fast. In the open area, the maximum communication distance is up to 305 meters, while in the more enclosed areas, the communication distance can also achieve 76 to 122 meters[13][14].

\section{RTK TERMINAL SYSTEM}

\section{A. Consisting of RTK Terminal System}

The RTK terminal system referred by this article especially means the compass and GPS satellite positioning RTK terminal system with WiFi access function. A complete RTK terminal system is mainly composed of two parts, hardware and software systems.

System hardware includes six portions which are instrument enclosure, interface, control board, satellite antenna, satellite positioning engine,power supply and etc. Among them, the instrument cases are consist of two parts. One is lightemitting diode, such as power pack 、 satellite 、 difference、Bluetooth、 Network and so on, the other is ABS of Power button, and the internal of it has solid metal stents; The interface owns WiFi antenna SMA interface, USB, Bluetooth, RS232, HDMI and other common interfaces; In the first phase, the control board use low-power MINI ATX H66 architecture board, whose memory is $4 \mathrm{G}$, i3 processor, 64G solid-state storage and operating system uses Windows7; Satellite antenna uses built-in GPS and the system of Compass 6 dual-frequency system; Satellite positioning engine uses a dual system OEM board, built-in GPS and Compass Channel 6; Power Modules use DC-DC module combination, power capacity uses lithium-ion battery pack which is combined into a 5200mAh from 18650. As shown in Fig.1,Fig.2.

System software includes serial debugging software and RTK positioning software.

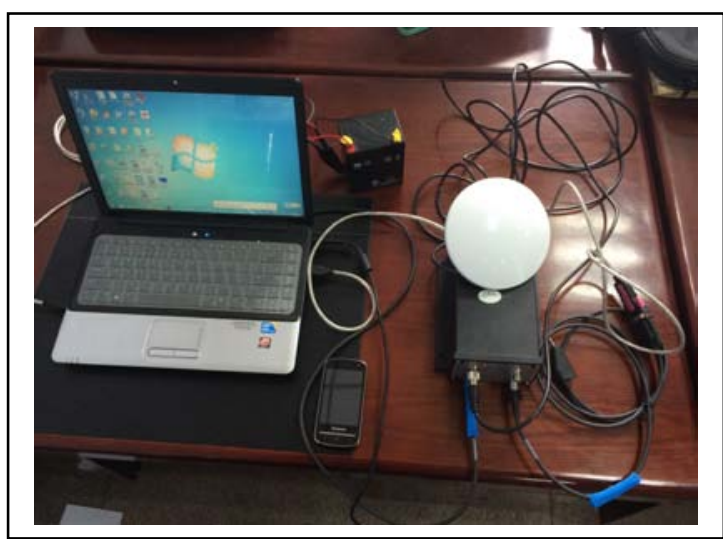

Fig. 1. The Actual Picture of RTK

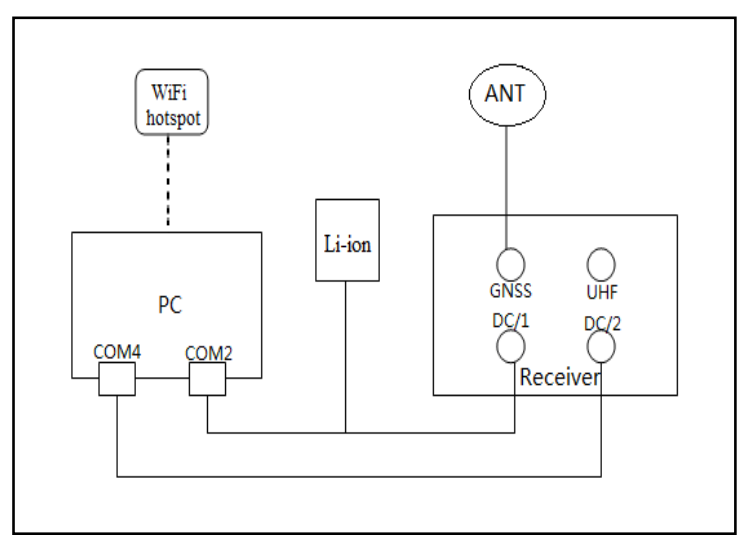

Fig. 2. The Ichnography of RTK

\section{B. Work Flow of RTK Terminal}

Satellite positioning receiver with the capabilities of $\mathrm{WiFi}$ wireless networking and the function of Compass and dynamic precision differential GPS(RTK) access to the local continuous tracking reference station(CORS) or user-built single reference station network through WiFi access to the surrounding Wi-Fi hotspot or temporary Wi-Fi hotspot constructed by the 3G mobile communications, then achieve real-time RTK Compass and the carrier phase correction parameters of GPS satellite positioning, achieves centimeter-level positioning. As shown in Fig.3 


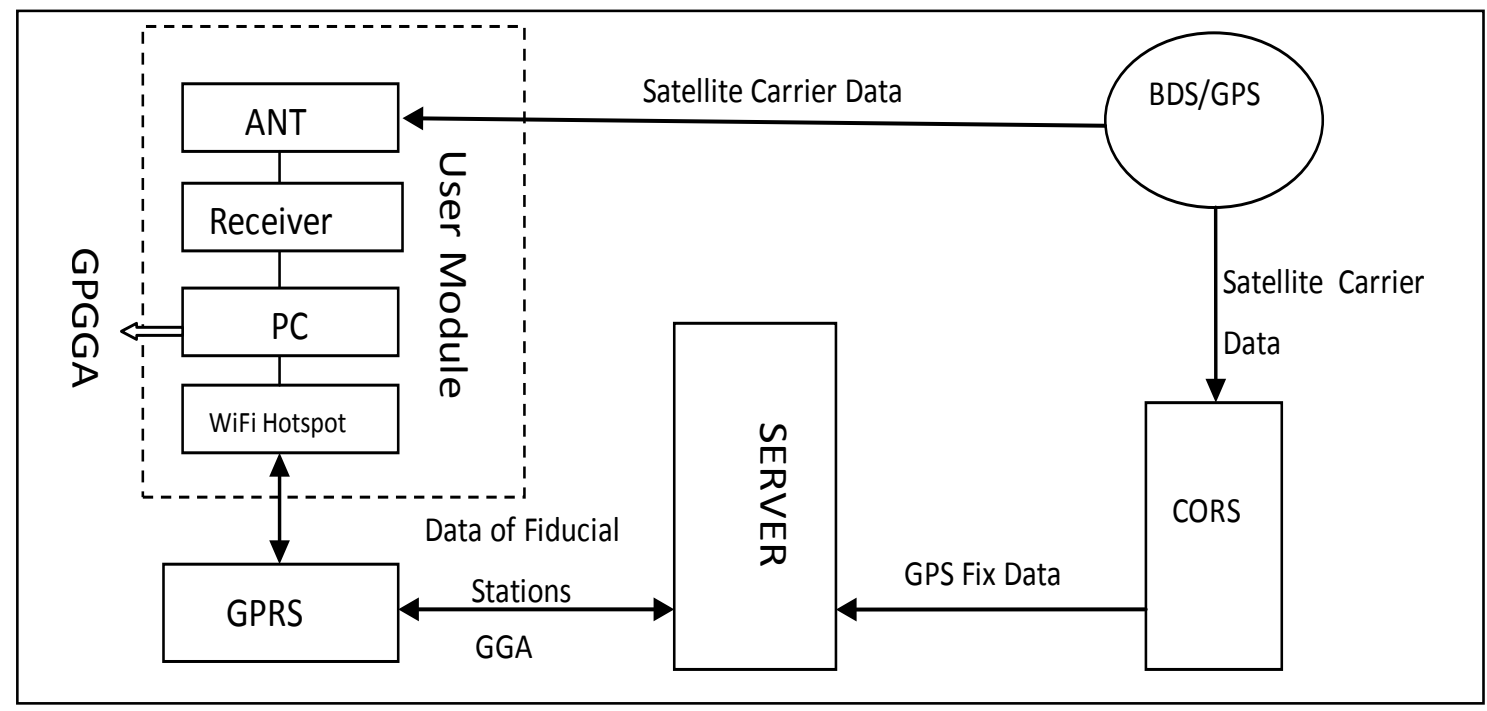

Fig. 3. Work Flow of RTK Receiver

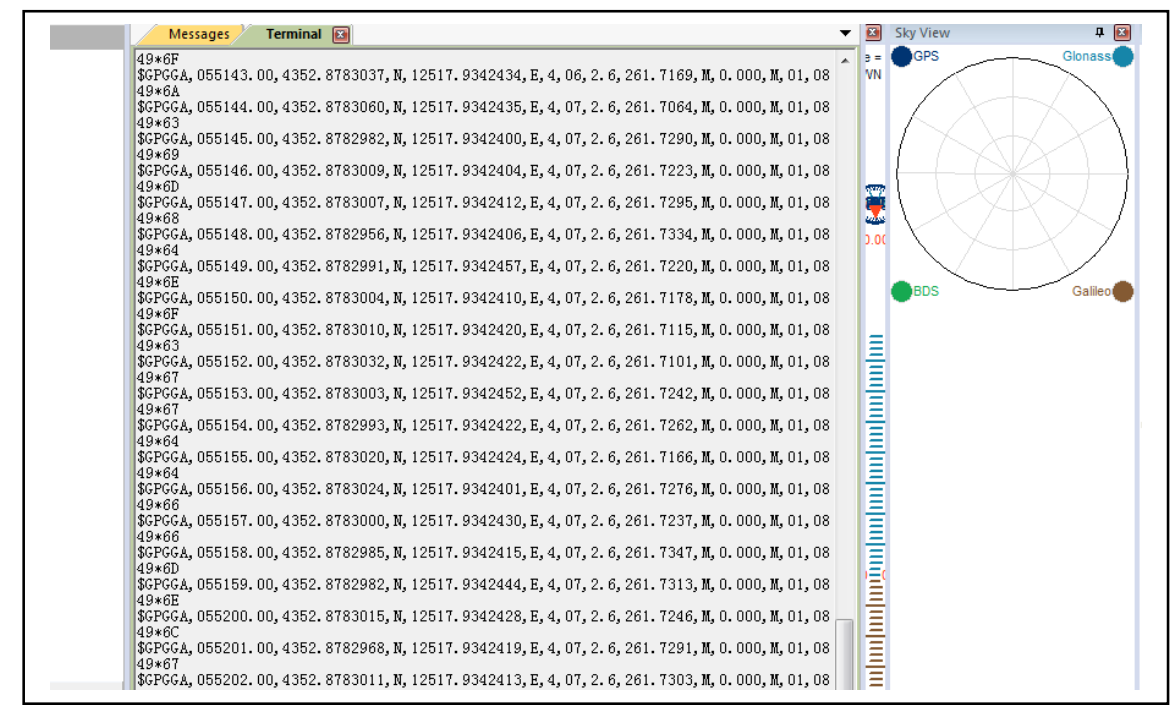

Fig. 4. Measured Data

\section{TEST AND ANALYSIS}

\section{A. Debugging Process}

First, the Wireless Fidelity hotspot devices should be configured to JLCORS which then be started up, while the receiver and the computer are connected by using USB, and post number is modified. Input the control command one by one to get the WGS-84 geodetic coordinates and to output data format of GPGGA after starting serial port debugging software. When the number of " $\mathrm{E}$ " is 4, the fixed solution is obtained. As shown in Fig.4

\section{B. Data Processing}

UTM projection universal transverse Mercator projection, the horizontal axis is cut conformal cylindrical projection, the central meridian projection length ratio is 0.9996. UTM projection is divided into 60 projection zones, from $180 \mathrm{~W}$ to $180 \mathrm{E}$, and the area is 6 degree. WGS-84 geodetic coordinates to the UTM projection conversion relations are as follows[15][16].

Rectangular coordinate formula,

$$
\begin{aligned}
& x=0.9996\left[S+\frac{l^{2} N}{2} \sin B \cos B+\right. \\
& \left.\frac{l^{4}}{24} N \sin B \cos ^{3} B\left(5-t^{2}+9 \eta^{2}+4 \eta^{4}\right)+\ldots\right] \\
& y=0.9996\left[l N \cos B+\frac{l^{3} N}{6} \cos ^{3} B\left(1-t^{2}+\eta^{2}\right)\right. \\
& \left.+\frac{l^{5} N}{120} \cos ^{5} B\left(5-18 t^{2}+t^{4}\right)+\ldots\right]
\end{aligned}
$$

Convergence of meridian formula, 


$$
\gamma=l \sin B+\frac{l^{3}}{3} \sin B \cos ^{2} B\left(1+3 \eta^{2}\right)+\ldots
$$

Where $S$ is the meridian arc length from the equator, length to central meridian ratio is 0.9996 , the true value formula of rectangular coordinate,

$$
\begin{aligned}
\mathrm{y}_{r}=\mathrm{y}+50000(\text { East of axis }) \\
\mathrm{y}_{r}=500000-\mathrm{y}(\text { Wast of axis }) \\
\mathrm{x}_{r}=10000000-\mathrm{x} \quad(\text { Southern Hemisphere }) \\
\left.\mathrm{x}_{r}=\mathrm{x} \quad \text { ( Northern Hemisphere }\right)
\end{aligned}
$$

The results of UTM projection coordinates are shown in Table 1.

TABLE I. UTM PROJECTED COORDINATES

\begin{tabular}{cccc}
\hline Epoch & $\mathbf{x ( m )}$ & $\mathbf{y}(\mathbf{m})$ & $\mathbf{h}(\mathbf{m})$ \\
\hline 1 & 4821842.819 & 676106.216 & 262.568 \\
2 & 4821842.819 & 676106.214 & 262.566 \\
3 & 4821842.819 & 676106.214 & 262.566 \\
4 & 4821842.822 & 676106.213 & 262.570 \\
5 & 4821842.820 & 676106.216 & 262.565 \\
6 & 4821842.820 & 676106.214 & 262.567 \\
7 & 4821842.820 & 676106.214 & 262.564 \\
8 & 4821842.819 & 676106.214 & 262.567 \\
9 & 4821842.818 & 676106.214 & 262.565 \\
10 & 4821842.817 & 676106.214 & 262.556 \\
\hline
\end{tabular}

\section{Accuracy Analysis}

\section{1) Statistics}

Calculate the average value of unknown quantity by multigroup observed value,

$$
\begin{gathered}
x=\frac{\sum_{i=1}^{N} x_{i}}{N} \\
y=\frac{\sum_{i=1}^{N} y_{i}}{N} \\
h=\frac{\sum_{i=1}^{N} h_{i}}{N}
\end{gathered}
$$

\section{Calculations are}

$\mathrm{x}=4821842.819 \mathrm{~m} ; \mathrm{y}=676106.216 \mathrm{~m} ; \mathrm{h}=262.563 \mathrm{~m}$ 。

\section{2) Diagram Display}

The following broken line chart depicted the relationship the $\mathrm{x}, \mathrm{y}$, h coordinates of the multi group observation data and the average value, of which the blue line and the pink one respectively represent the average of multi group observations and each epoch observations. From the chart, each epoch observations basically distributed into the two sides of the average value. The jump errors of $\mathrm{X}$ direction , Y direction , $\mathrm{H}$ direction respectively were no more than $0.6 \mathrm{~cm}, 1.0 \mathrm{~cm}$ and 3.0cm(Fig.5,Fig.6,Fig.7,Fig8).

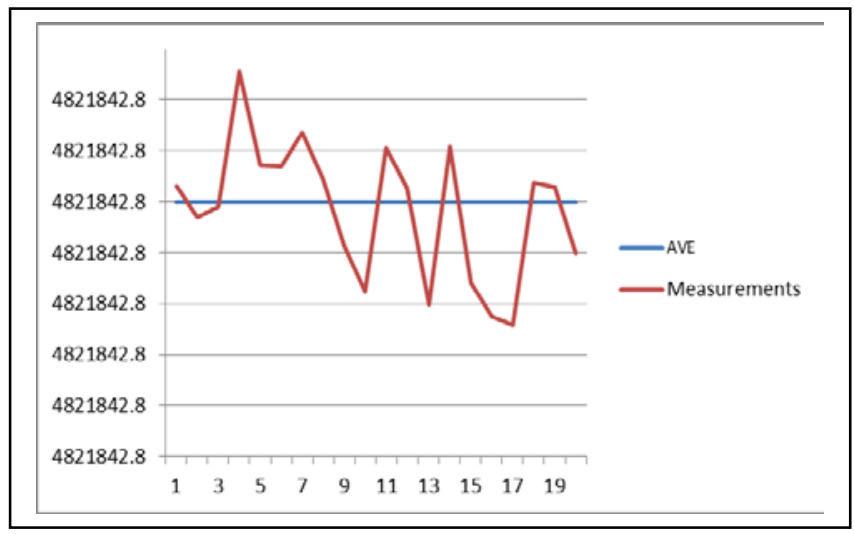

Fig. 5. Line Chart of $x$ Coordinate

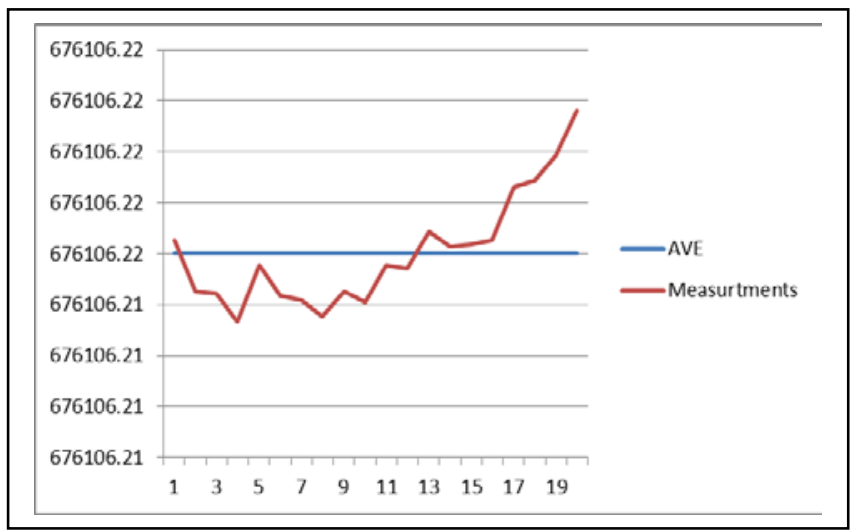

Fig. 6. Line Chart of y Coordinate

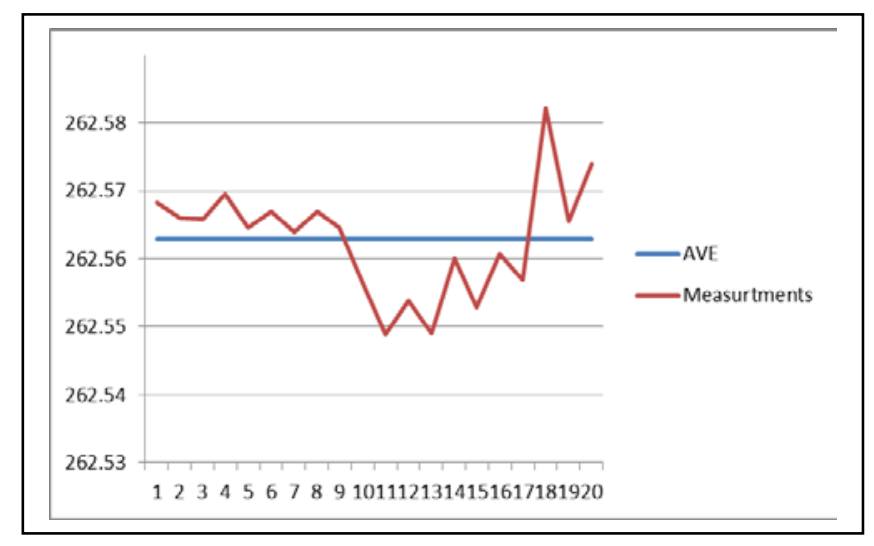

Fig. 7. Line Chart of h Coordinate 


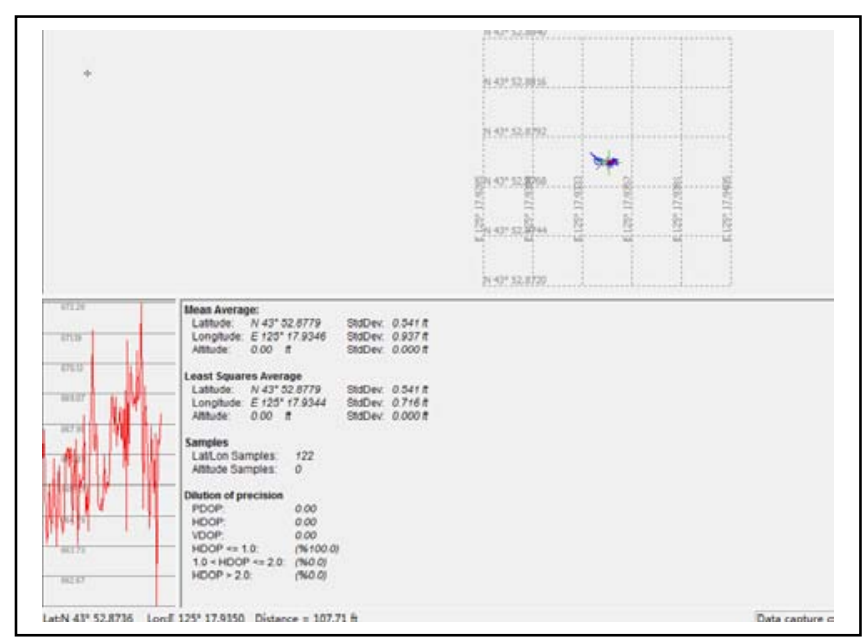

Fig. 8. Measurement Accuracy

\section{3) Inner Precision Rating}

Inner precision is used to evaluate the accuracy as the index. The arithmetical mean of all observations which were received from fixed measuring points was regarded as the maximum likelihood values. Make a difference between each observation and the maximum likelihood values one by one and calculate difference square sum. The followed formula will be utilized to calculate the internal accuracy of three directions( $\mathrm{x}, \mathrm{y}, \mathrm{h})$.

$$
\sigma= \pm \sqrt{[\Delta \Delta] /(n-1)}
$$

where $\sigma$ represents inner precision, $n$ means the total number of the same observations, $\Delta$ means the difference of observation and the maximum likelihood values.

Using the appeal formula, the accuracy of the $\mathrm{x}$ direction is $0.2 \mathrm{~cm}$, the direction of $\mathrm{y}$ is $0.3 \mathrm{~cm}$, and the accuracy of $\mathrm{h}$ is $0.9 \mathrm{~cm}$. It can be obtained that the accuracy of the plane is higher, the precision is higher than the $0.3 \mathrm{~cm}$, the height precision is relatively low, the precision is higher than $1 \mathrm{~cm}$, which is about $1 / 5$ of the plane precision, but all the accuracy is centimeter level, and the stability is very high.

\section{CONCLUSION}

The design of Big Dipper RTK receiver based on Wireless Fidelity hotspot wireless communication data link will be used to RTK difference and union of complex communication means and communication protocol which be globally accepted to improve the consistency, generality, compatibility on a global scale. The paper design to receive real time carrier phase correction parameter, analyze Data and evaluate precision by using the method that the RTK receiver is connected to JLCORS station. Finally, the result of centimeter level real- time positioning is obtained, and the design requirements of the Big Dipper RTK receiver based on WIFI hotspot are basically realized.

\section{ACKNOWLEDGMENT}

This study is supported by College of Geoexploration Science and Techology, Jilin University.

\section{REFERENCES}

[1] YU Xiao-long,HU Xue-kui,”GPS RTK Technique:Merits,Demerits and Perspective ,” J.Bulletin of Surveying and Mapping,no.4.2007,pp.39-41.

[2] ZHANG Zhen-jun,XIE Zhong-hua,FENG Chuan-yong.”The Method Study of RTK Survey Precision Assessing ,’J. Bulletin of Surveying and Mapping,no.1.2007,pp.26-28.

[3] Chen Wen-zhou. "The Research and Application of WiFi Technology,” J. Data Communications,no.2.2008,pp.14-17.

[4] Wang Wei,Shi Ting-yu,Zhang Zhi-quan. “The Application and Prospect of CORS System ,’J .Urban Geotechnical Investigation \& Surveying,no.3.2010,pp.45-47.

[5] WANG Shijin;BEI Jinzhong;LI Dehai;ZHU Huizhong.” RealTimeJKinematic Positioning Algorithm of GPS/BDS,”J. Geomatics and Information Science of Wuhan University,no.5.2014,pp.621-625.

[6] R Odolinski; P J G Teunissen; D Odijk.” Combined GPS + BDS for short to long baseline RTK positioning,"J. Measurement Science \& Technology,vol.26,no.4.pp.1-16.

[7] Gur S, Sikka SC, Abdel-Mageed AB, et al. "Imatinib Mesylate (Gleevec) Induces Human Corpus Cavernosum Relaxation by Inhibiting Receptor Tyrosine Kinases (RTKs): Identification of New RTK Targets,”J.Urology,vol.82,no.3.2013,pp.11-16.

[8] Xie Shi-jie, Xi You-geng. "Characteristics and Error Analysis of RTK," J. Engineering of Surveying and Mapping, no.2.2002,pp.34-37.

[9] WEI Rui-juan,LI Xue-jun,REN Wei-cheng,Nie Rong-jian. “A Study of Single-based Station CORS Construction and Its Application ,’J. Bulletin of Surveying and Mapping ,no.6.2010,pp.23-26.

[10] WU Mengqi;GUO Jiming;ZHOU Changzhi;ZHANG Haiping;FENG Yantong.” Existing Problems for CORS Systems and the Solutions,”J. Existing Problems for CORS Systems and the Solutions,no.5.2015,pp.54-56.

[11] Wang Jing-hua,Wu Yu-chen. "JLCORS Application in Water Conservancy Engineering ,"J. Jilin Water Resources,no.7.2012,pp.31-33.

[12] Tang Si-min, “Research of The WiFi Technology and Its Application ,”J. Fujian Computer,no.10.2009,pp.59-61.

[13] Zeng Lei,Zhang Hai-feng,Hou Wei-yan. "Design and Implement of WiFi Wireless Measurement and Control Network ,’J. Electrical Measurement \& Instrumentation,no.7.2011,pp.81-84.

[14] Zhao Man;Hou Xiu-mei. "Design of mine signal transceiver based on WiFi technology ,’J. Industry and Mine Automation,no.72014,pp.5-8.

[15] Lian Bao-wang,Zhang Yi,Li Yong.” Journal of Northwestern Polytechnical University,”J. Journal of Northwestern Polytechnical University,no.s.1999,pp.170-174.

[16] Zhou Chao-xian, Fang Zhi-feng, Yu Cai-hong, Zhang Yun-guo, Gao Ying-bo, Yan Dan-chen, Yang Qiang. "UTM projection and Gauss-Krüger projection and their conversion ,’J. Geology and Exploration,vol.49,no.5.2013, pp. $882-889$. 